\title{
Preparation of a Novel Sol-Gel Molecularly Imprinted Polymer with Dummy Template for On-Line Solid-Phase Extraction of Patulin from Apple Juice Samples
}

\author{
Afshin Rajabi Khorrami*, Yalda Pasandideh \\ Department of Chemistry, Karaj Branch, Islamic Azad University, Karaj, Iran
}

Received: May 05, 2016; Accepted: June 20, 2016; Published: June 27, 2016

*Corresponding authors: Afshin Rajabi Khorrami, Department of Chemistry, Karaj Branch, Islamic Azad University, Karaj, Iran, E-mail: a-khorrami@kiau.ac.ir

\begin{abstract}
Patulin is a toxic chemical produced by a number of certain species growing on any mouldy or rotten fruits specially apple. A novel, sensitive and simple on-line Solid-Phase Extraction (SPE) based on sol-gel Molecularly Imprinted Polymer (MIP) with isatin as dummy template coupled to HPLC was developed and validated for separation and determination of patulin from apple juice samples. In optimum conditions good linearity was achieved from 0.02 to 0.5 $\mu \mathrm{g} \mathrm{ml}-1$ with relative standard deviations of about $1.98 \%$. The Limit of Detection (LOD) of was $0.011 \mu \mathrm{g} \mathrm{ml}-1$ and the recoveries of spiked apple juice samples were more than $88 \%$. The recovery results showed that the proposed method not to be affected by the presence of matrix substances and can be applied successfully for the analysis of patulin in apple juice samples without any special pre-treatment.
\end{abstract}

Keywords: Sol-gel; Molecularly imprinted polymer; Patulin; Online solid phase extraction; Apple juice.

\section{Introduction}

Patulin (Figure 1a) with the IUPAC name of (4-hydroxy-4Hfuro $[3,2-c]$ pyran-2(6H)-one) is a toxic chemical produced by a number of certain moulds. Patulin contamination is of main concern with apples spoiled by moulds such as Penicillium expansum. Although apples and apple-based products tend to be the major sources of patulin, but any mouldy or rotten fruits, vegetables, cereals, and other foods could contain this toxin [1]. This compound was studied at first as a compound possessing wide spectrum antibiotic properties, but next researches demonstrated the toxicological properties at patulin such as mutagenic, immunotoxic, neurotoxic and causing adverse effects on the gastrointestinal tract [2]. The presence and the extent of patulin contamination in processed apple products can consider as a parameter for the quality of the fruits used for processing and the safety of the product. The European Union (EU) set levels of patulin at $50 \mu \mathrm{gg}^{-1}$ for apple juices and apple juice ingredients, $25 \mu \mathrm{g} \mathrm{kg}^{-1}$ for solid apple products (such as apple compote, apple puree, etc.) and $10 \mu \mathrm{g} \mathrm{kg}^{-1}$ in apple-based products for infants and young children (European Commission Regulation, 1881/2006). Joint FAO/WHO Expert Committee on Food Additives (JEFCA)<smiles>O=C1C=C2C(=CCOC2O)O1</smiles>

(a)<smiles>O=C1Nc2ccccc2C1=O</smiles>

(b)
Figure 1: Structural formula of patulin (a) and isatin (b).

established a provisional maximum tolerable daily intake (PMTDI) of $0.4 \mu \mathrm{g} \mathrm{kg}^{-1}$ body weight/day based on a no observed effect level (NOEL) of $43 \mu \mathrm{g} \mathrm{kg}^{-1}$ body weight/day (WHO, 1995).

Several analytical methods for the determination of patulin have been published in recent years, including thin-layer chromatography (TLC) [3]. Liquid-Liquid Extraction (LLE) [4]. Liquid Chromatography-Mass Spectrometry (LC-MS) [5]. HighPerformance Liquid Chromatography-UV Detection (HPLC-UV) [6]. Gas Chromatography-Mass Spectrometry (GC-MS) [7,8]. Also, an electrochemical method has been used for patulin determination in apple juice samples [9]. GC-MS methods are not satisfactory for routine analysis, because they require derivatization of patulin before starting analysis. Liquid-liquid Extraction with final LC-UV detection is the most widely used and has been validated as an AOAC International official method [10].

In the most of the above works, a pre-concentration or cleanup step is required before analysis to achieve the necessary levels of sensitivity. Nowadays, Solid-Phase Extraction (SPE) is one of the most valuable enrichment techniques. This technique has so many advantages compared to Liquid-Liquid Extraction (LLE) such as simplicity, shorter extraction time, better recovery, higher enrichment factor, lower cost, lower consumption of organic solvents and easy automation. However, typical SPE sorbents lack selectivity and this constitutes a problem when a selective extraction from a complex matrix has to be performed [11]. 
In the recent years, Molecularly Imprinted Polymers (MIPs) have been employed for the preparation of materials with specific molecular recognition properties to enhance the molecular selectivity in solid-phase extraction. The advantages of MIP-SPE over other selective sorbents such as specificity and stability have made it as one of the most important techniques for selective sample concentration in biological, pharmaceutical, and environmental fields $[12,13]$.

MIPs are mainly prepared using vinyl and acrylic monomers or through the condensation of Organically Modified Silanes
(ORMOSILs) giving rise to hybrid sol-gel or xerogel materials [1416]. Molecularly imprinted sol-gel materials have two important advantages over traditional imprinted polymers. First, they are synthesized under mild conditions, not requiring heat or radiation to polymerize. This aspect offers important advantages when dealing with thermal and photosensitive template molecules. Second, the majority of imprinting technology, demonstrated to date, involves the synthesis of MIPs in organic monomer-solvent systems. Moreover, most polymerization systems lack of true water compatibility [17]. But unlike organic MIPs, sol-gels are<smiles>CCO[Si](OCC)(OCC)OCC</smiles>

Tetraethoxysilan

(TEOS)

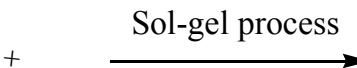<smiles>O=C1Nc2ccccc2C1=O</smiles>

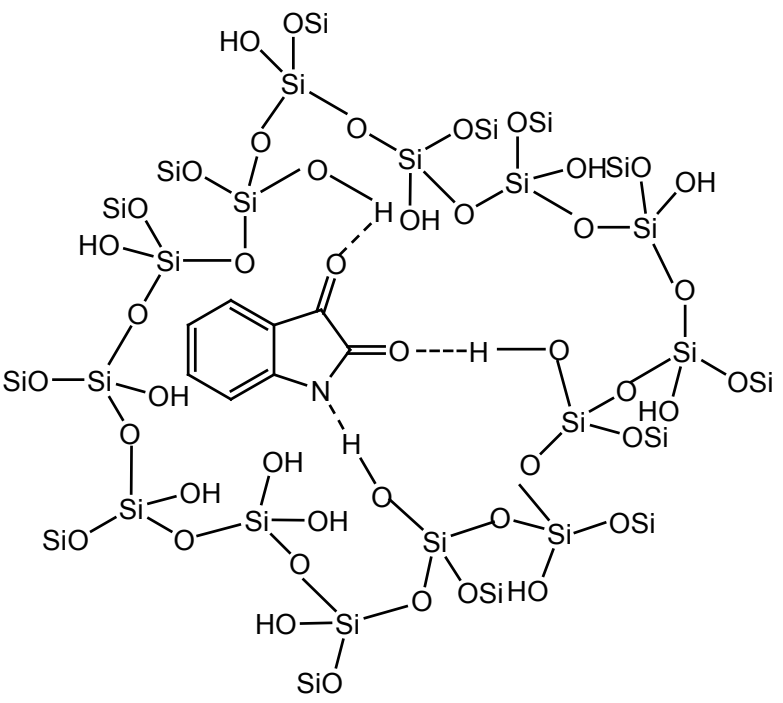

Isatin (Template)

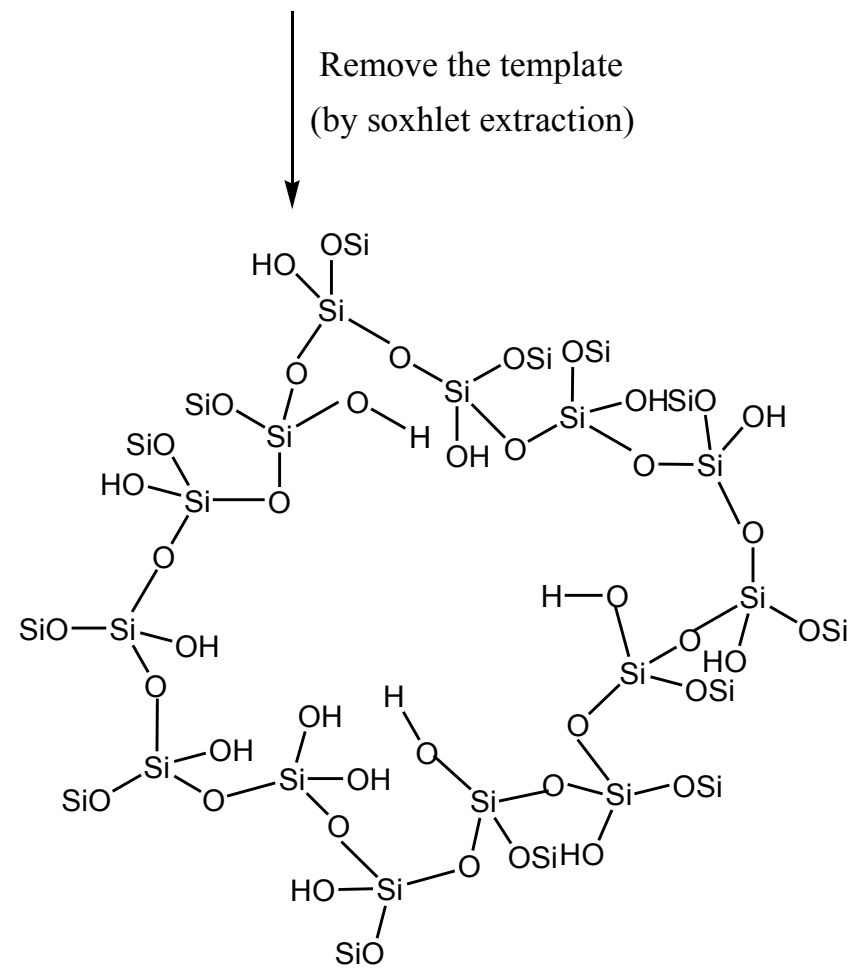

Figure 2: A possible schematic representation of dummy template molecular imprinting sol-gel polymerization. 


\section{synthesized in aqueous/alcoholic mixtures as solvent media.}

On the other hand, a significant problem which often encountered in MIP-SPE of trace analytes is the "bleeding" of template trapped within the polymer network. A trace amount of the template used in the pre-polymer mixture remains bounded in the polymer even after thorough washing of the polymer [18]. An elegant solution to this problem is the utilization of an analogous dummy template during polymerization. With this approach, any leakage of the dummy template will not interfere with the analysis of target molecule. Dummy templates also have the advantage of being lower cost than the target and typically less reactive with the polymerization reagents. This strategy has been successfully employed to extract several analytes from different matrices [4,10,19-23].

The polymers that synthesized by sol-gel methods are very suitable for online SPE [24,25]. During on-line SPE-HPLC, same as offline SPE, the sample is loaded onto a SPE cartridge where the analytes are retained while unretained matrix components went to the waste tank. Then, the analytes are automatically transferred from the SPE cartridge to the analytical column for chromatographic separation. On-line SPE minimizes the need for manual operations such as evaporation and reconstitution, which are usually required in off-line SPE and sources of error in the analysis of biofluids [26].

The objective of this work is to synthesize a novel molecularly imprinted sol-gel polymer by combining imprinting technique with a sol-gel process using is a tin as a dummy template. This compound was selected because of its structural similarity with patulin and reasonable price. Figure $1 \mathrm{~b}$ shows the structural formula of isatin. Then, the prepared phase was used to develop a sensitive and rapid analytical technique for the selective separation and analysis of traces of patulin in apple juice samples.

\section{Experimental}

\section{Chemicals and materials}

Patulin, 5-(Hydroxymethyl) Furfural (HMF) and Tetraethylorthosilicate (TEOS) were purchased from SigmaAldrich (St. Louis, USA). Is a tin as template, all solvents in HPLC grade including Tetrahydrofuran (THF), methanol, ethanol, acetonitrile, ethyl acetate, hydrochloric acid and other chemicals were from Merck (Darmstadt, Germany).

Stock standard solution of patulin has been prepared by dissolving $5 \mathrm{mg}$ of pure crystalline patulin in $250 \mathrm{ml}$ of ethyl acetate. Stock solution was stored at $-20^{\circ} \mathrm{C}$. Working standard solutions were prepared by appropriate dilution of stock solution with double distilled water acidified with hydrochloric acid at $\mathrm{pH}$ $=4.0$. All working solutions were freshly prepared prior to each experiment, wrapped in aluminum foil and stored at $4^{\circ} \mathrm{C}$.

\section{Apparatus}

High-performance liquid chromatography experiments were performed with a Perkin-Elmer chromatographic system (CA, USA) that was equipped with a binary pump model 200, ultraviolet detector model 200 and a Rheodyne six port switching valve. The Total Chrom software was applied to acquire and process spectral and chromatographic data from the UV detector. The SPE mini column was a $8 \mathrm{~mm}$ i.d. $\times 20 \mathrm{~mm}$ long stillness steel column with appropriate frits at both ends.

All separations were achieved on an analytical C18 column (Machery-Nagel, $5 \mu \mathrm{m}, 4.6 \mathrm{~mm}$ i.d. $\times 25 \mathrm{~cm}$ long, $5 \mu \mathrm{m}$ pore size) with a mobile phase flow rate of $1.0 \mathrm{~mL} \mathrm{min-1}$ under isocratic conditions at room temperature. The wavelength of detector was fixed at $276 \mathrm{~nm}$ in all experiments.

\section{Sol-gel synthesis}

One m mol $(0.147 \mathrm{~g})$ of isatin and $800 \mu \mathrm{l}$ of concentrated hydrochloric acid were dissolved in de-ionized water. After 30 min, $27 \mathrm{ml}$ TEOS was added to the mixture. The precursor kept in an oil bath at 60 for 48 hours. The product was grounded and sieved, through 25 and $50 \mu \mathrm{m}$ sieves. Then, the is a tin molecules were removed from sol-gel polymeric network by repeated soxhlet extraction with solvents including water, methanol, acetonitril and ethyl acetate until the template was not detected by UV-Vis-spectrophotometer. A possible schematic representation of this process is shown in figure 2. A Non-Imprinted Sol-Gel Polymer (NIP) was synthesized simultaneously using the same protocol, in the absence of the isatin.

\section{On-line SPE-HPLC experiments}

To evaluate the procedure, the cylindrical SPE stainless steel minicolumn was packed with $1 \mathrm{~g}$ synthesized sol-gel polymeric sorbent. The minicolumn was placed in the sample-loop position of the six-port injector valve of HPLC. A schematic diagram for on-line SPE pre-concentration and analysis of patulin is shown in figure 3. When the HPLC injector valve was in the load position, $2 \mathrm{ml}$ of working solutions were injected manually into the mini column by a syringe with a flow rate of $2.0 \mathrm{ml} \mathrm{min}-1$. The patulin was adsorbed and unwanted solutions went to the waste tank (Figure 3a). Then, the valve was switched from the 'load' to the 'inject' position. In the inject position the patulin adsorbed on the SPE minicolumn were eluted in the back flush mode by the HPLC mobile phase at a flow rate of $1.0 \mathrm{ml} \mathrm{min-1}$ into the chromatographic separation column (Figure 3b). Finally, the valve was switched to the loading position to clean the SPE minicolumn with methanol and de-ionized water for the next cycle, while the analytes were separated in the chromatographic separation column.

\section{Apple juice analysis}

The blank juice sample was prepared from fresh apples and stored at 4 until assay. The absence of patulin was checked by official AOAC method. The apple juice samples were purchased from a local market. Firstly, all juices were centrifuged for $10 \mathrm{~min}$ at 10,000 rpm and were filtered with $0.45 \mu \mathrm{m}$ Nylon membran filters. Then, the clear supernatants were acidified with hydrochloric acid at $\mathrm{pH}=4.0$. The samples were spiked with appropiate amounts of patulin working solution to estimate recoveries. The analysis was proceded as described in the previous section. 

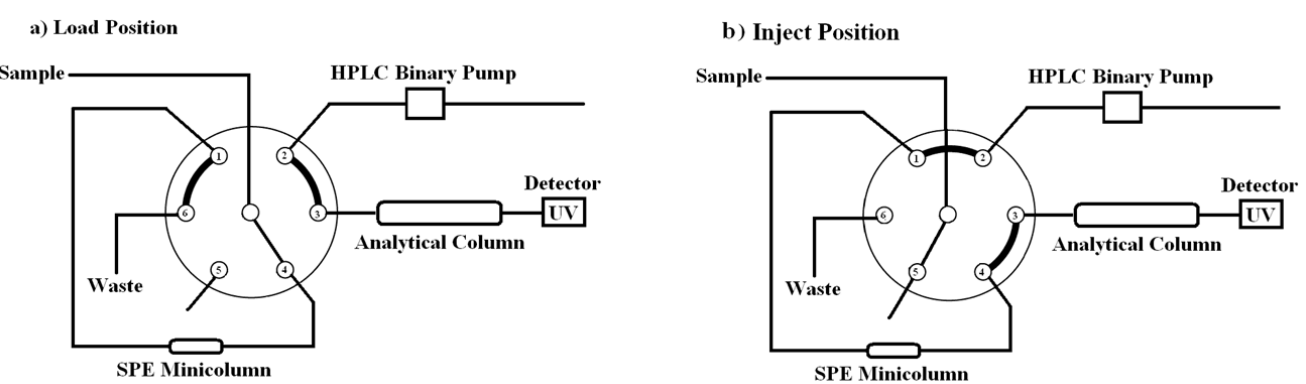

Figure 3: Schematic diagram of the on-line SPE coupled to HPLC.

\section{Results and Discussion}

\section{Experimental optimization}

One of the most effective parameters in adsorption, selectivity and pre-concentration procedures is the $\mathrm{pH}$ of samples that can change the ionization form of analytes. The pH effect of the sample solution on patulin recovery was studied using $0.05 \mu \mathrm{g}$ ml-1 standard solutions in a $\mathrm{pH}$ range from 2 to 8 . The $\mathrm{pH}$ of solutions was adjusted with dilute $\mathrm{HCl}$ or $\mathrm{NaOH}$. According to the results in figure 4 , the maximum recovery was observed at $\mathrm{pH}=$ 4.0. Therefore, this $\mathrm{pH}$ was adjusted for further studies.

5- Hydroxy Methyl Furfural (HMF) is the main interference during patulin determination and generally prevents a reliable quantitative determination [26]. HMF and patulin exhibits similar chromatographic characterizations owing to their chemical structures. Hence, optimization of eluent was a huge problem, because it must have been strong enough to desorbed patulin from SPE minicolumn, and also suitable mobile phase to separate the patulin from the other compounds in analytical column. For this purpose several mobile phases such as methanol, acetonitrile, ethanol, ethyl acetate, THF, water and different combinations of them were examined. The highest recovery and separation of patulin from other compounds was performed using H2O/THF $(80: 20 \mathrm{v} / \mathrm{v})$. Thus this ratio was used as eluent/mobile phase for further experiments.

The influence of loading flow rate, that indicates the effective contact time between patulin and the sorbent, was also studied. This parameter was evaluated in the flow rate range of 1.0 to $4.0 \mathrm{~mL} \mathrm{~min}-1$. The results in figure 5 . Shows that the

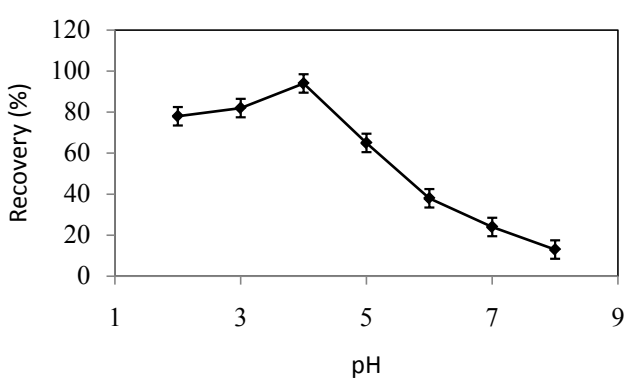

Figure 4: The effect of $\mathrm{pH}$ on the recovery of $0.05 \mathrm{\mu g} \mathrm{ml}^{-1}$ patulin using the proposed method.

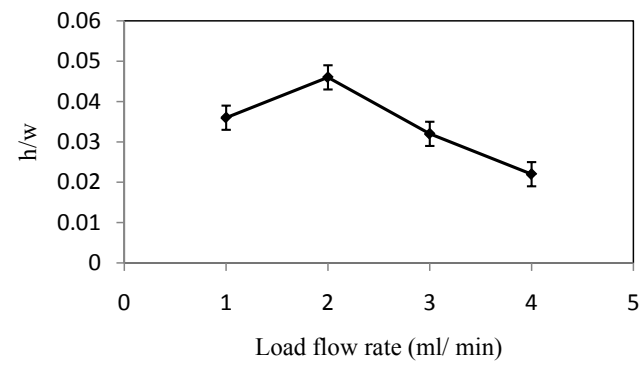

Figure 5: The effect of loading flow rate on peak heights to width ratio of $0.05 \mu \mathrm{g} \mathrm{ml}^{-1}$ patulin using the proposed method.

chromatographic peaks height/width ratio of patulin increased as the loading flow rate decreased from 4.0 to 1.0 mLmin-1, and the best loading flow rate was achieved at $2.0 \mathrm{~mL} \mathrm{~min}-1$. Thus, for next experiments, loading flow rate of $2.0 \mathrm{~mL} \mathrm{min-1}$ was chosen.

In the next attempt, the effect of elution flow rate was studied in order to find required time for desorption of patulin from minicolumn. This parameter was tested in the flow rate range of 0.2 to $1.2 \mathrm{~mL} \mathrm{min-1.} \mathrm{As} \mathrm{it} \mathrm{is} \mathrm{shown} \mathrm{in} \mathrm{figure} 6$, the chromatographic peak heights to width ratio of patulin linearly increased as elution flow rate (desorption time) increased from 0.2 to $1.0 \mathrm{~mL} \mathrm{~min}-1$, and leveled off in 1.0 to $1.2 \mathrm{~mL} \mathrm{~min}-1$. So, elution flow rate of $1.0 \mathrm{~mL} \mathrm{~min}-1$ was selected for the next experiments.

After optimization of influencing parameters, the proposed method was applied to apple juice samples. The resulting chromatograms are shown in figure 7. Chromatogram of blank sample is shown in figure7a. The HMF and isatin peaks were eluted in less than five minutes. Then, the apple juice was spiked with patulin standard solution at $0.05 \mu \mathrm{g} \mathrm{ml}-1$. As it is shown in figure $7 \mathrm{~b}$, it was successfully separated from other compounds. To guarantee the lack of HMF interference, the sample solution was spiked at $0.2 \mu \mathrm{g}$ ml-1 level. Figure7c Shows the peaks of patulin and HMF were completely separated and no interference was observed.

\section{Imprinting effect}

In order to evaluate the presence of imprinted sites on the synthesized polymer, the Non-Imprinted Sol-Gel Polymer (NIP) packed in the minicolumn was employed. Then, standard patulin solution containing $0.05 \mu \mathrm{g} \mathrm{ml}-1$ of patulin in de-ionized water at 


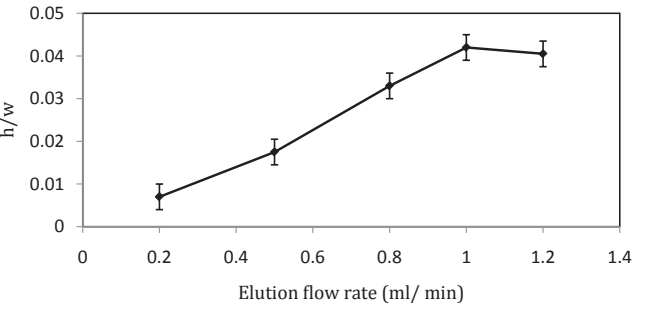

Figure 6: The effect of elution flow rate on peak heights to width ratio of $0.05 \mu \mathrm{g} \mathrm{ml}^{-1}$ patulin using the proposed method.

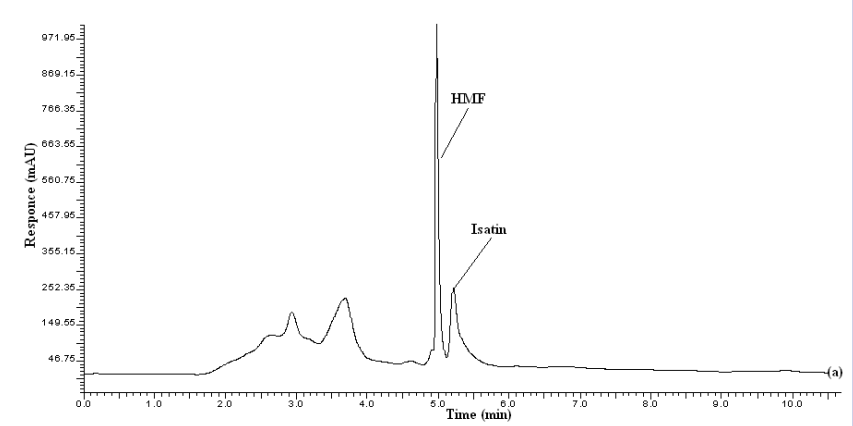

Figure 7a: Chromatograms of blank apple juice (a).

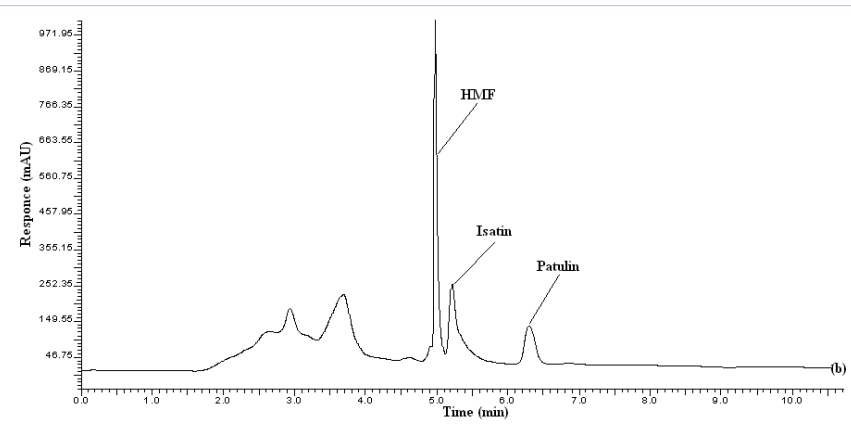

Figure 7b: Spiked sample with $0.05 \mu \mathrm{g} \mathrm{ml}^{-1}$ patulin (b).

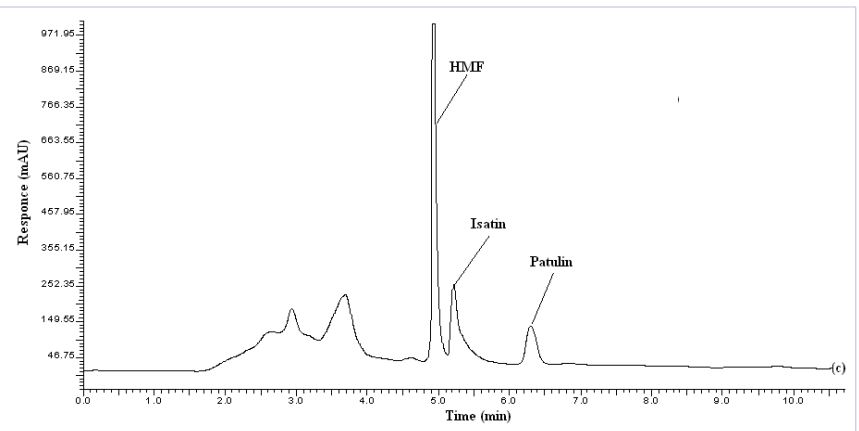

Figure 7c: Spiked sample with $0.05 \mu \mathrm{g} \mathrm{ml}^{-1}$ patulin and $0.2 \mu \mathrm{g} \mathrm{ml}^{-1} \mathrm{HMF}$ (c).

$\mathrm{pH}=4$ was loaded onto the SPE minicolumn at the flow rate of 2.0 $\mathrm{mL}$ min- 1 and continued by the proposed procedure. Comparison of the recoveries in figure 8 shows that the selectivity of imprinted polymer for patulin was significantly greater than non-imprinted one.

\section{Analytical figures of merit}

The analytical figures of merit for the present on-line SPEHPLC procedure were investigated under optimized conditions. The calibration curve for quantification was achieved by plotting peak areas versus the spiked concentration of patulin to real samples under optimum conditions. The obtained graph with equation $\mathrm{Y}=2 \times 107 \mathrm{X}-317212$ in figure 9 shows excellent linearity for patulin over a concentration range of 0.02-0.5 $\mu \mathrm{g} \mathrm{ml}-1$ with a Regression Coefficient (R2) of 0.999.

The Limit of Detection (LOD) and Limit of Quantification (LOQ) for six repeated analyses based on three and ten times of the signal to noise ratio of the baseline near the patulin peak were 0.011 and $0.039 \mu \mathrm{g} \mathrm{ml}-1$, respectively.

The intra-day and inter-day precision were calculated for five repeated runs of apple juices spiked at $0.05 \mu \mathrm{g} \mathrm{ml}-1$ and the results showed a Relative Standard Deviation (RSD) of $1.98 \%$ and $4.24 \%$, respectively.

\section{Application to commercial samples}

The proposed procedure was applied for determination of patulin in some commercial apple juice samples. The juices were spiked with 0.05 and $0.2 \mu \mathrm{g} \mathrm{ml}-1$ of patulin and analyzed by the developed online SPE-HPLC procedures were summarized in table 1. The excellent recoveries in all cases proved high ability of the developed procedure for determination of patulin in real samples.

\section{Conclusion}

In this study, a novel sol-gel molecularly imprinted polymer has been synthesized by combining molecular imprinting

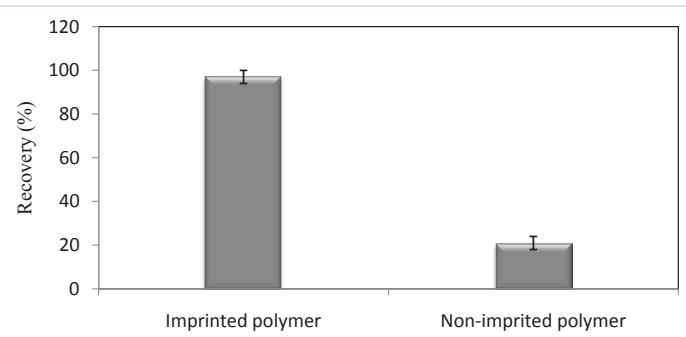

Figure 8: The imprinting effect of imprinted polymer versus non-imprinted packed using the proposed method.

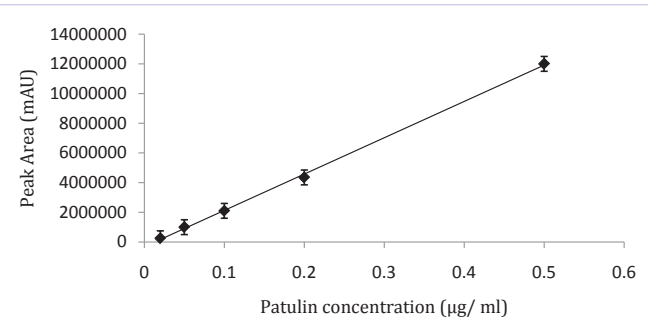

Figure 9: Calibration curve of patulin with different spike levels. 
Table 1: Recovery results of patulin from spiked apple juice samples using proposed online SPE-HPLC method.

\begin{tabular}{|l|l|l|l|}
\hline Sample & Added $\left(\boldsymbol{\mu g} \mathbf{~ m l}^{\mathbf{1}}\right)$ & Found $\left(\boldsymbol{\mu} \mathbf{~ m l}^{-\mathbf{1}}\right)$ & Recovery (\%) \\
\hline Sample 1 & 0 & 0.015 & - \\
\hline & 0.05 & 0.059 & 88.0 \\
\hline & 0.2 & 0.197 & 91.0 \\
\hline Sample 2 & 0 & 0.120 & - \\
\hline & 0.05 & 0.167 & 94.0 \\
\hline & 0.2 & 0.311 & 95.5 \\
\hline Sample 3 & 0 & 0.342 & - \\
\hline & 0.05 & 0.388 & 92.0 \\
\hline & 0.2 & 0.523 & 90.5 \\
\hline
\end{tabular}

technique with a sol-gel process with high affinity to patulin. Isatin was used as dummy template during polymerization process. The prepared sol-gel polymer was successfully applied to develop a selective on-line-SPE-HPLC procedure for preconcentration and determination of patulin from apple juice samples. The method showed a simple and rapid procedure to analysis patulin with satisfactory precision and recovery. The proposed method did not affect by matrix interferences in apple juice samples.

\section{References}

1. Oliveira RC, Bando É, Machinski Junior M. Interlaboratory optimization and validation of a method for patulin determination in grapes by thin-layer chromatography, Brazilian Journal of Microbiology. 2007;38:304-8.

2. Claude B, Morin P, Bayoudh S, de Ceaurriz J. Interest of molecularly imprinted polymers in the fight against doping. Extraction of tamoxifen and its main metabolite from urine followed by highperformance liquid chromatography with UV detection. J Chromatogr A. 2008;1196-1197:81-8. Doi: 10.1016/j.chroma.2008.05.022.

3. Andersson L, Paprica A, Arvidsson T. A highly selective solid phase extraction sorbent for pre concentration of sameridine by molecularly imprinting. Chromatographia. 1997;46(1-2):57-62.

4. MacDonald S, Long M, Gilbert J, Felgueiras I. Liquid chromatogtaphic method for determination of patulin in clear and cloudy apple juices and apple puree: collaborative study. JAOAC Int. 2000;83(6):1387-94.

5. Rajabi Khorrami A, Rashidpur A. Development of a fiber coating based on molecular sol-gel imprinting technology for selective solid-phase micro extraction of caffeine from human serum and determination by gas chromatography/mass spectrometry, Anal Chim Acta. 2012;727:20-5. Doi: 10.1016/j.aca.2012.03.048.

6. Wang XJ, Xu ZL, Feng GL, Bing NC, Yang ZG. Molecular imprinted membranes for the recognition of lovastatin acid in aqueous medium by a template analogue imprinting strategy. J Membr Sci. 2008;313(12):97-105.

7. Han DM, Fang GZ, Yan XP. Preparation and evaluation of a molecularly imprinted sol-gel material for on-line solid-phase extraction couple with high performance liquid chromatography for the determination of trace pentachlorophenol in water samples. J Chromatogr A. 2005;1100(2):131-6.

8. Wu RN, Han FL, Shang J, Hu H, Han L. Analysis of patulin in apple products by liquid-liquid extraction, solid phase extraction and matrix solid phase dispersion methods: a comparative study. Eur Food Res Technol. 2009;228(6):1009-14.

9. Sook Cho M, Kim K, Seo E, Kassim N, Barthalomew Mtenga A, Shim WB, et al. Occurrence of Patulin in Various Fruit Juices from South Korea: An Exposure Assessment. Food Sci. Biotechnol. 2010;19(1):1-5.

10. Theodoridis G, Kantifes A, Manesiotis P, Raikos N, TsoukaliPapadopoulou H. Preparation of a molecularly imprinted polymer for the solid-phase extraction of scopolamine with hyoscyamine as a dummy template molecule. J Chromatogr A. 2003;987(1-2):103-9.

11. Matsui J, Fujiwara K, Takeuchi T. Atrazine-selective polymers prepared by molecular imprinting of trialkylmelamines as dummy template species of atrazine. Anal Chem. 2000;72(8):1810-3.

12. Rupp HS, Turnipseed SB. Confirmation of patulin and 5-hydroxymethylfurfural in apple juice by gas chromatography/mass spectrometry. J AOAC Int. 2000;83(3):612-20.

13. AOAC Official Methods of Analysis of AOAC International, Liquid Chromatographic Method for Determination of Patulin in Clear and Cloudy Apple Juices and Apple Puree: Collaborative Study, Food chemical contaminants. 2000;83(6):1387-94.

14. Li TJ, Chen PY, Nien PC, Lin CY, Vittal R, Ling TR, et al. Preparation of a novel molecularly imprinted polymer by the sol-gel process for sensing creatinine. Anal Chim Acta. 2012;711:83-90. Doi: 10.1016/j. aca.2011.10.031.

15. Kubo T, Hosoya K, Watabe Y, Ikegami T, Tanaka N, Sano T, et al. Oncolumn concentration of bisphenol A with one-step removal of humic acids in water. J Chromatogr A. 2003;987(1-2):389-94.

16. Tabata S, Iida K, Suzuki J, Kimura K, Ibe A, Saito K. A quantification and confirmation method of patulin in apple juice by GC/MS. Shokuhin Eiseigaku Zasshi. 2004;45(5):245-9.

17. Barkai-Golan R (Editor), Paster N (Editor). Mycotoxins in Fruits and Vegetables. Academic Press (Elsevier). First edn. 2008:261-70.

18. MacDonald S, Long M, Gilbert J, Felgueiras I. Liquid chromatogtaphic method for determination of patulin in clear and cloudy apple juices and apple puree: collaborative study. JAOAC Int. 2000;83(6):1387-94.

19. Díaz-García ME, Badía-Laíño R. Molecular imprinting in sol-gel materials: recent developments and applications. Microchim Acta. 2005;149(1-2):19-36.

20. Ellwanger A, Berggren C, Bayoudh S, Crecenzi C, Karlsson L, Owens PK, et al. Evaluation of methods aimed at complete removal of template from molecularly imprinted polymers. Analyst. 2001;126(6):784-92.

21. Theodoridis G, Kantifes A, Manesiotis P, Raikos N, TsoukaliPapadopoulou H. Preparation of a molecularly imprinted polymer for the solid-phase extraction of scopolamine with hyoscyamine as a dummy template molecule. J Chromatogr A. 2003;987(1-2):103-9.

22. He J, Wang S, Fang G, Zhu H, Zhang Y. Molecularly imprinted polymer on-line solid-phase extraction coupled with high performance liquid chromatography-UV for the determination of three sulfonamides in pork and chicken. J Agric Food Chem. 2008;56(9):2919-25. Doi: 10.1021/jf703680q.

23.León González Z, Ferreiro-Vera C, Priego-Capote F, de Castro MD. Targeting metabolomics analysis of the sunscreen agent 2-ethylhexyl4(N,N-dimethylamino)benzoate in human urine by automated online solid phase extraction-liquid chromatography-tandem mass spectrometry with liquidchromatography-time-of-flight/mass spectrometry confirmation. J.ChromatogrA. 2011;1218(20):3013-21. 
Doi: 10.1016/j.chroma.2011.03.045.

24. Rajabi Khorrami A, Taherkhani M. Synthesis and Evaluation of a Molecularly Imprinted Polymer for Pre-concentration of Patulin from Apple Juice. Chromatographia. 2011;73(1): 151-6.
25. Qiao F, Sun H, Yan H, Ho Row K. Molecularly Imprinted Polymers for Solid Phase Extraction. Chromatographia. 2006;64(11/12):626-34.

26. Gupta R, Kumar A. Molecular imprinting in sol-gel matriz. Biotechnol Adv. 2008;26(6):533-47. Doi: 10.1016/j.biotechadv.2008.07.002. 